\title{
Arte contemporáneo: temporalidad, territorialización y circulación
}

\author{
Jonathan Feldman \\ CONICET-IIAC/UNTREF, IIEAC-UNA (Argentina) \\ jonfeld@gmail.com
}

RESUMEN: Este artículo da cuenta de diferentes definiciones teóricas del arte contemporáneo y sus características, recurriendo a los enfoques de Giorgio Agamben, Terry Smith, Boris Groys y Julieta Aranda, para, a continuación, poner en relación estas posiciones con la noción de territorialización esbozada por Deleuze y Guattari (1985), y así posar la mirada sobre tres niveles de territorialidad que atraviesan las definiciones de arte contemporáneo: local, regional y global. Esto permite, finalmente, vincular estos niveles a los modos de circulación y exhibición del arte contemporáneo para generar discursos sostenidos sobre la base de las instituciones y los mercados artísticos. Se hace también una breve descripción de los casos de dos instituciones porteñas que permiten comprobar estos niveles de territorialidad y las formas de circulación del arte contemporáneo.

PALABRAS CLAVE: Arte contemporáneo; Territorialización; Glocalización; MAMBA; CCBA; Argentina.

\section{Contemporary Art and Territorialisation. The State of the Art}

ABSTRACT: This work intends to provide a view of different theoretical definitions of contemporary art and its characteristics. For this purpose it studies the approaches of Giorgio Agamben, Terry Smith, Boris Groys and Julieta Aranda. Secondly, this article seeks to relate these positions with the notion of territorialisation provided by Deleuze and Guattari (1985) to focus on three levels of territoriality: local, regional and global. Finally this will allow the linking of these levels with the ways contemporary art circulates and the ways in which these phenomena are exhibited to generate discourses about contemporary art that are sustained by art institutions and markets. Lastly, this article analyzes two cases of institutions in Buenos Aires to offer a view of these levels of territorialisation and the ways in which contemporary art circulates through them.

KEYWORDS: Contemporary Art; Territorialisation; Glocalisation; MAMBA; CCBA; Argentina.

Recibido: 13 de abril de 2017 / Aceptado: 23 de junio de 2017.

\section{Introducción}

El presente trabajo se propone realizar un breve estado de la cuestión respecto de algunas definiciones de arte contemporáneo que están en el centro de la discusión del campo artístico actual. Para ello primero se posará la mirada sobre el concepto de era contemporánea presentada por Giorgio Agamben (2009) y su relación con el arte contemporáneo. Luego se analizarán algunas posiciones respecto del arte contemporáneo desde, al menos, dos perspectivas: su relación con el proyecto artístico de la Modernidad, y la vinculación con el tiempo y la temporalidad. En este sentido, se intentará dar cuenta de las perspectivas que se adoptan para generar discursos respecto del arte contemporáneo y la forma en las cuales estos son apropiados, sostenidos y discutidos por las instituciones y los mercados por los que circulan. Además se trabajará sobre las formas con las que el arte contemporáneo opera y tematiza cuestiones ligadas a los procesos de territorialización, siguien-

Cómo citar este artículo: FELDMAN, Jonathan: "Arte contemporáneo: temporalidad, territorialización y circulación», Boletín de Arte-UMA, n. ${ }^{\circ} 38$, Departamento de Historia del Arte, Universidad de Málaga, 2017, pp. 87-96, ISSN: 0211-8483, DOI: http://dx.doi.org/10.24310/BoLArte.2017.v0i38.3222 
do el concepto presentado por Deleuze y Guattari (1985), y la manera en la cual se ponen en juego los niveles local, regional y global en la contemporaneidad, especialmente, en relación al arte contemporáneo. Se pondrá el foco en la formación de redes de circulación artística y en los nuevos vínculos entre los actores que participan en los procesos de producción de las artes contemporáneas, y los modos en las cuales se exhiben.

Para ello se analizará, por último, el caso de dos instituciones porteñas (el Museo de Arte Moderno en Buenos Aires y el Centro Cultural de España en Buenos Aires) y su relación con estos tres niveles territoriales, con el objeto de identificar las maneras en las que operan en el mapa artístico en estos tres niveles y comparar algunas características y tipos de funcionamiento.

\section{Algunas posiciones respecto del arte contemporáneo}

En un intento de abordar lo que llamó la «era contemporánea», Giorgio Agamben (2009: 1) caracterizó la contemporaneidad como «una relación singular con el propio tiempo, que adhiere a éste y, a la vez, toma distancia». El autor intentaba dar cuenta de lo que significa ser contemporáneo a partir de definir un vínculo de los sujetos con el tiempo y de posar la mirada sobre las sombras de una época, de no dejarse deslumbrar por sus luces para ver sus escondites. Para Agamben, contemporáneo es aquel que puede, a pesar de estar observando esas sombras, percibir la luz que lo persigue pero nunca lo alcanza. En otras palabras, la contemporaneidad permite relaciones temporales diversas y particulares, una suerte de multiplicación de capas de tiempo, una anacronía que permite unir el presente con lo pasado y lo futuro, traer el pasado al presente y a su vez volver pasado lo presente, y presente lo futuro. Ser contemporáneo, entonces, implicaría vivir el tiempo presente en una escala donde los polos son lo ocurrido y lo todavía por ocurrir, y tener la capacidad de unir temporalidades: traer el pasado al presente, acercar el presente al futuro.

En cuanto a las artes, se podría pensar en lo dicho por Agamben para hablar de aquello que propone este tipo de vínculos temporales, es decir, aquel arte en el cual el presente es todo lo que hay, pero a su vez ese presente está asociado inexorablemente al pasado y al futuro. En estas diferentes relaciones temporales, lo contemporáneo puede ser pensado en el campo de las artes desde un devenir: lo contemporáneo es aquello que está sucediendo, lo que es simultáneo al momento. Tal vez sería posible afirmar que el arte contemporáneo estimula, al estar en continuo presente, la multiplicidad, al permitir diferentes presentes. En este sentido la relación que establece con el tiempo es múltiple y expansiva.

Este tipo de vínculo temporal se puede analizar en relación a la finalización del proyecto teleológico de la Modernidad, cuyo relato suponía una cierta verdad y un destino: en el arte contemporáneo, además de la inexistencia de grandes relatos, se observa un futuro incierto, un presente contingente que no afirma más que algo es de una manera, sino que algo se puede ver de cierta manera. Así, la multiplicación de presentes pareciera ser una característica del arte contemporáneo, así como su privilegiada relación con el ahora y aquello que deviene, que sucede. Este tipo de acercamiento al arte contemporáneo se trabajará más detenidamente en el desarrollo posterior de este artículo

Sin embargo, el arte contemporáneo es reconocido por algunos autores como tal desde, al menos, los sesenta. Probablemente sea más acertado, en este sentido, hablar de operaciones del arte contemporáneo, pues permite analizar los comportamientos de las artes desde sus modos de hacer, desde sus formas de producción. Si se toma en cuenta esta posición, Julieta Aranda (2010) propone que la categoría del arte contemporáneo es algo que, si bien en desarrollo, ya ha tomado formas tales que merezcan una historización, aunque por algún motivo se escapa de ello. Tal vez uno de los motivos para ello sea, justamente, su multiplicidad: mientras que las vanguardias modernas competían diacrónicamente por el lugar de la verdad declarada (es decir, por ser el arte), las producciones contemporáneas ofrecen, al sustraerse de un gran relato, una posibilidad sincrónica.

Visto desde otro punto de vista, el constante devenir del arte contemporáneo hace que se pueda volver tanto novedad como extinción, pues aquello que parece un problema artístico deja de serlo casi inmediatamente, y lo mismo sucede con los modos de producción de ciertos artistas. Pamela Lee (cit. en Foster, 2010: 3) denomina al problema de la historicidad del arte contemporáneo como «síndrome del blanco móvil», es decir, un constante movimiento de 
condiciones que no permite clasificación más que provisoria. Si se piensa más en esto último, el carácter provisorio del arte contemporáneo, se puede observar, además, que las prácticas artísticas contemporáneas se han vuelto contingentes, lo que contrasta con las prácticas del arte moderno: ya no existe una necesidad de totalización, aquello que el proyecto de la Modernidad puso como horizonte, sino que se trata de la presentación de una posibilidad, de un punto de vista. Esto mismo trae a primer plano la necesidad de volverse distintivo, lo que a su vez se traduce en una gran heterogeneidad.

Es esta misma heterogeneidad la que explica lo que en principio pareciera ser una desjerarquización de los estilos artísticos en la contemporaneidad: la versión totalizante ha sido reemplazada por diversas versiones particularizantes, lo que antes era señal de la presencia del arte hoy está en jaque. Al respecto de esto, sin embargo, se debe aclarar que tanto las instituciones artísticas como los artistas en sí siguen siendo marca de artisticidad.

Si se vuelve sobre las definiciones de arte contemporáneo, pareciera ser que todo arte actual es contemporáneo y, en este sentido, lo contemporáneo funcionaría como un gran paraguas bajo el cual se englobaría toda producción artística del momento. Así es como las preguntas respecto de su definición se observan como anacrónicas, pues «es precisamente a través de su aparente auto-evidencia que dejan de ser problemáticas y empiezan a ejercer su influencia en formas escondidas» (Aranda, Wood, Vidloke, 2010: 1).

Esta fuerza expansiva del arte contemporáneo permite que acepte la incorporación de una gran diversidad de modos de producción y tipos de expresiones, y que esquive definiciones y demarcación de un adentro y afuera del campo por la invisibilidad de sus límites (Aranda, Wood, Vidloke, 2010: 2). Esta misma invisibilidad hace que el campo artístico se vea como inagotable y capaz de fagocitar cualquier fenómeno social hacia el interior de sus fronteras. Pero en realidad se trata más bien de un sistema cuyas reglas parecen estar en constante mutación, y eso abona a su carácter provisorio pues dificulta, entre otras cosas, una lectura estilística, material o disciplinar dominante.

Por otro lado, lo contemporáneo en sí es visto por algunos autores como Boris Groys (2009: 3) como constituido por la duda e indecisión, de reconsideración de los proyectos modernos. Para este tipo de proyectos se necesitaba un fin, una aspiración, una promesa de futuro. Pero el arte contemporáneo funciona de otro modo: las colecciones permanentes se reemplazaron por las exhibiciones temporarias, los proyectos artísticos ponen el acento sobre lo presente y lo pasado, y los artistas trabajan con aspiraciones casi instantáneas. En palabras del autor: «El presente ha dejado de ser un punto de transición del pasado al futuro, convirtiéndose en un lugar de permanente re-escritura tanto del pasado como del futuro» (Groys, 2009: 4).

Si se retoma la perspectiva del tiempo, Terry Smith define el arte contemporáneo a razón de su multiplicidad: "con tempus comenzó a utilizarse, y continúa en uso, debido a su capacidad de hacer referencia a una multiplicidad de relaciones entre el ser y el tiempo» (Smith, 2012: 19). Esto significa, por un lado, que las diferentes expresiones artísticas toman el tiempo como tema y producen entonces obras que obligan a una experiencia temporal y, por otro lado, que la recepción origina relaciones diferentes con el tiempo: una instalación ofrece un vínculo de temporalidad distinta que una proyección o una performance.

Pero también, lo que Smith parece afirmar es que la propia diversidad estilística y de modos de producción que para el autor caracteriza al arte contemporáneo supone una diversidad temporal, pues el presente se duplica como el punto de vista y la existencia temporal se vuelve también múltiple y coexistente.

\section{Niveles de territorialidad: local, regional, global}

Otro aspecto reconocido como característico del arte contemporáneo se relaciona con la circulación y aspectos de la territorialidad, entendida como una conformación de prácticas sociales y políticas. En este sentido los territorios pueden ser vistos como dinámicos y en conformación: el planteo de Deleuze y Guattari sostiene que «no hay territorio sin un vector de salida del territorio, y no hay salida del territorio, desterritorialización, sin que al mismo tiempo se dé un esfuerzo para reterritorializarse en otro lugar, en otra cosa» (en entrevista con Claire Parnet, 1996: s/p). Así se pueden pensar los territorios como procesos «de dominio (político-económico) o de apropiación (simbólico-cultural) del espacio por grupos humanos, en un conjunto complejo y variado ejercicio de poder(es)» (Haesbaert, 2011: 11), que 
son múltiples y se constituyen a través de diversas relaciones entre individuos, sistemas y geografías.

Una de las características del mundo contemporáneo pareciera ser el borramiento de las fronteras y la transnacionalización. Con el desarrollo de la economía globalizada y la expansión de las comunicaciones, muchos sostienen que la conformación de territorios se ve afectada debido a su incapacidad de delimitación geográfica. De modo que se ponen en dificultades las soberanías nacionales y se proponen vínculos globales que generan otras unidades y niveles territoriales. En general, las categorías que sostienen estas nuevas unidades en la contemporaneidad son lo global, lo regional y lo local. El primer término proviene del mundo financiero, en tanto la acumulación de capital produjo movimiento y reconstrucción de territorios que finalmente se cristalizaron en la transnacionalización del capital y de los mercados financieros. La apertura de la lógica centro-periferia se puede observar, por ejemplo, en la aparición de centros económico-financieros en lugares como Kuala Lumpur o en la construcción arquitectónica de ciudades como Dubai, que la ligan simbólicamente a los históricos centros financieros como Wall Street.

Con respecto a los otros dos niveles, lo local se relaciona con un nivel bajo de desplazamiento humano y, por lo tanto, se considera local aquello que no supera una extensión de 500 kilómetros. En este sentido lo local se basa en la proximidad y su acción parece más concentrada. Lo regional, por su parte, propone la salida de las formas nacionales de la modernidad para componer otras unidades, sub y supranacionales, como la región patagónica en Argentina, o la Comunidad Europea en el viejo continente. En este sentido, el alcance de esta categoría es más amplio que el de lo local, suponiendo una extensión geográfica mayor.

Sin perjuicio de las definiciones de estos tres niveles, todos ellos están presentes en un territorio dado, ya que las relaciones que se conforman parecieran demarcar una multiplicidad presente en todos los procesos de territorialización que se traduce, también, en multiplicidad identitaria (Deleuze y Guattari, 1994: 14; Haesbaert, 2011: 15-17). De modo que, se podría afirmar, lo global, lo regional y lo local como categorías territoriales permiten, por un lado, una pista de ciertos modos de producción del arte contemporáneo y, por otro lado, un acercamiento a la cuestión de su circulación. Respecto de lo primero, las artes contemporá- neas parecieran darle un lugar prominente a las prácticas territoriales como forma de producción y la intervención de estos territorios como base para sus obras. Así se pueden encontrar ejemplos como trabajos en ciertas comunidades y desarrollo de soluciones a problemas de acceso a bienes y servicios culturales, la construcción de series escultóricas en espacios públicos con la participación de la ciudadanía en su producción (como Culture in Action, un proyecto de mediados de los noventa que se desarrolló en la ciudad de Chicago), la renovación del espacio público con intervenciones artísticas o incluso afectando el paisaje (como sucedió en Documenta 13 con el Museo de la Muerte de Adrián Villar Rojas), la señalización de problemas históricos, socio-financieros o ambientales, o incluso el redescubrimiento de ciertas zonas geográficas o de lugares de importancia local o regional; como sucede con las Expediciones a Puerto Piojo, en La Boca. Se podría entonces afirmar que las prácticas artísticas contemporáneas se perfilan como territoriales, en tanto se ocupan de modificar los territorios sobre los cuales actúan. Esto también tiene gran relación con los fenómenos de movilidad y migración que se producen en la contemporaneidad, especialmente en Europa. Este efecto se podría vincular a la concepción múltiple de la identidad y de lo territorial de Deleuze, quien no piensa lo identitario como algo unívoco sino como una multiplicidad que incluye al «Otro».

Respecto del segundo punto, el discurso acerca del arte contemporáneo suele poner el énfasis en su carácter global y lo vincula, por un lado, con la mayor circulación y movilidad de artistas, curadores y públicos y, por otro lado, con la conformación de un mercado verdaderamente transnacional. Ambos procesos fueron facilitados por el desarrollo tecnológico y comunicacional y por los movimientos de capitales, y se observan en fenómenos como las bienales y las ferias de arte, cada vez más frecuentes y más extendidas a geografías antes ignoradas, lo que pareciera afirmar el carácter reticular del arte contemporáneo. El alcance expandido de las herramientas tecnológicas y la gran movilidad que caracteriza a los artistas contemporáneos producen cambios en las relaciones de visibilidad de ciertas producciones artísticas, regionales o locales, dentro del panorama global. Sin embargo, debe haber también un reconocimiento de los verdaderos alcances de esta globalización: «En realidad, la globalización no es tan global como parece, o, para ser exactos, es mucho más global para algunos que para otros, la mayoría» (Mosquera, 2010: 33). 
Esta afirmación se relaciona con el alcance desigual de una herramienta como internet, así como una tendencia a la occidentalización de las producciones, pues justamente la distribución irregular de la tecnología resulta, para el autor, en una formación reticular no igualitaria, que parte desde los centros para instalarse luego en la periferia.

Otro punto de vista es el esbozado por Cuauhtémoc Medina (2009: 2), quien identifica dentro de la desigualdad de distribución del poder cultural un avance del sur en la imaginación global. Sin embargo este avance está para el autor incompleto o sesgado, porque si bien existe un desarrollo de infraestructuras de arte contemporáneo en ciudades y regiones periféricas o emergentes, la necesidad de participación de un mercado global hace que las referencias y metáforas regionales o locales se deban insertar dentro de una lógica de circulación y diseminación artística global dominante, en clave de contemporaneidad (Medina, 2010: 73).

Esta preponderancia de lo contemporáneo sobre la procedencia de las producciones artísticas hace que no se pueda seguir entendiendo el arte del sur como desfasado o atrasado respecto del arte del norte, y la institucionalización a la cual se refiere Medina se relaciona más bien con las formas de circulación y producción del arte (aunque no se deba entender por ello que el arte contemporáneo es constitutivamente homogéneo). Esta presencia del arte contemporáneo a su vez local, regional y global permite pensar en las maneras en las cuales los espacios expositivos y las instituciones artísticas producen relatos que integran estos tres niveles. Es interesante reflexionar sobre esto en relación a las producciones y los espacios argentinos, en los cuales se podría observar las formas de interacción de los fenómenos artísticos locales con aquellos de la región sudamericana o latinoamericana, y su inserción dentro del circuito global.

\section{Dos espacios porteños}

Antes de mencionar y relevar dos instituciones de Buenos Aires, es importante realizar una cartografía de los espacios de arte contemporáneo en la ciudad que permita dar cuenta del contexto en el cual desarrollan sus propuestas las instituciones con las cuales se ejemplificará luego, a saber, el Museo de Arte Moderno de Buenos Aires y el Centro Cultural de España en Buenos Aires.
Se podría comenzar mencionando la gran apertura de espacios dedicados a exhibir arte contemporáneo en las últimas dos décadas, y la necesidad de actualizar propuestas de algunos museos de gran tradición para adecuarlos a los públicos contemporáneos; es el caso, por ejemplo, del Museo Nacional de Bellas Artes, que a partir de 2004 comienza a sufrir una serie de transformaciones programáticas y curatoriales tendentes a atraer nuevos públicos. En este sentido, en 1996 abrió en La Boca la Fundación PROA, un espacio dedicado al arte internacional y financiado por la corporación Tenaris que alberga exhibiciones de gran escala y generalmente itinerantes, y también posee un espacio (que se comparte con la cafetería) de arte contemporáneo específicamente.

En el año 2001, en pleno estallido de la crisis socioeconómica más grave de la historia argentina, se inauguró el Museo de Arte Latino Americano en Buenos Aires (MALBA), con obras provenientes de la colección de Eduardo Constantini y ayuda para la adquisición de obra complementaria procedente de fundaciones e inversores privados. Además de las salas dedicadas a la colección de arte latinoamericano se realizan exhibiciones temporarias de artistas nacionales e internacionales, con un programa muy enfocado en el arte contemporáneo.

Es interesante que en ambos casos las instituciones mencionadas fueran puntapiés para la organización -a lo largo más de una década y media- de circuitos expandidos. En La Boca, por ejemplo, se creó en 2012 el denominado «Distrito de las Artes» (un proyecto del Gobierno de la Ciudad de Buenos Aires que ofrece beneficios impositivos y financieros a proyectos culturales que se radiquen en las zonas de La Boca y San Telmo, con el fin de promover nuevos circuitos artísticos y agregar valor a las zonas relegadas por años), con galerías como POPA, Isla Flotante, Barro, Walden y La Verdi, todas dedicadas con exclusividad al arte contemporáneo y que exhiben obras de artistas tanto emergentes como consagrados, principalmente locales pero también de otras regiones. En Recoleta, por otra parte, se organizó un circuito que incluye entre otras a las galerías RolfArt, Miranda Bosch, María Calcaterra, La Ruche de Jorge Mara, Pasto, Henrique Faria BA y Rubbers (una galería tradicional y antigua). En estas galerías se expone tanto arte moderno como contemporáneo, aunque al igual que en el otro circuito también se presentan artistas emergentes y consagrados, locales e internacionales. 
Por último, un tercer y novedoso circuito de arte contemporáneo se localiza en Villa Crespo, que logró nuclear propuestas de galerías como Ruth Benzacar, Slyzmud, Arte $x$ Arte, Experiencia Hiedra, Nora Fisch y Hache. Se trata de espacios de arte contemporáneo, en algunos casos con vinculación a programas curatoriales y con presencia de residencias artísticas.

En relación a esta breve cartografía de los espacios de arte contemporáneo en Buenos Aires es que se ubican los casos que interesan a este trabajo: el MAMBA y el CCEBA. La selección se relaciona con dos cuestiones fundamentales: en primer lugar, la diversidad de características que presentan estas instituciones en cuanto a que son, respectivamente, un museo y un centro cultural; en segundo lugar, las visiones institucionales y, por lo tanto, los programas curatoriales que presentan de acuerdo a sus intereses y misiones.

El Museo de Arte Moderno de Buenos Aires fue creado en 1956 y su primer director fue el crítico de artes, Rafael Squirru. Uno de los principales objetivos del museo en sus comienzos se relacionó con posicionarse como un lugar de vanguardia, que presentara las producciones más actuales del país e integrara, no solamente artes visuales, sino además fotografía, diseño y otras disciplinas. La primera de sus exposiciones fue flotante, ya que el museo no poseía edificio propio y Squirru generó una exhibición de pintores argentinos que itineró, en el buque Yapeyú, por 22 ciudades del mundo. A lo largo de los años el Museo incrementó su colección a base de donaciones (la más importante realizada por Ignacio Pirovano) y adquisiciones que privilegiaron el arte argentino pero incorporaron, además, obras de artistas extranjeros. En la década de 1960 inauguró la Primera Exposición Internacional de Arte Moderno y su imagen se consolidó mundialmente como un referente del arte moderno tanto local como internacional. En 1986 el Museo comenzó a funcionar en su edificio actual, que solía ser la fábrica de la tabaquera Nobleza Picardo, en el barrio de San Telmo, y su programa se basó en sostenerse como referente global del arte moderno y contemporáneo argentino e internacional.

Respecto de su misión, es definida por las autoridades del espacio como:

Difundir y promover las manifestaciones del Arte Moderno y Contemporáneo (...) El Moderno también tiene como misión reflejar la multiplicidad de tendencias de la vida cultural (...) proponiendo un constante debate en torno al concepto de museo, a la historia del arte tradicional y a la figura de un nuevo espectador, considerado como un sujeto activo y político".

Hacia finales de la década de los noventa, tiempo en el cual el país estaba sumido en una crisis socioeconómica severa, con altos índices de desocupación e indigencia, la política de adquisición del museo se vio interrumpida y su función social se volvió casi predominante. Hacia el estallido de diciembre de 2001 y durante los siguientes dos o tres años el público del museo se constituyó principalmente por ciudadanos que intentaban escapar del calor de la crisis sin gastar mucho dinero². Luego de su refacción (entre 2005 y 2010) y algunas exhibiciones de artistas contemporáneos argentinos (entre ellas Últimas Tendencias II, en 2012) se comenzaron a modificar los programas expositivos para abandonar progresivamente el enfoque moderno y se consolida una propuesta de arte contemporáneo con la llegada de Victoria Noorthoorn a la dirección del museo en 2013, quien plantea la necesidad de asegurar la permanencia y prominencia del MAMBA en los circuitos artísticos contemporáneos de la ciudad.

Se podría decir que desde su concepción, y tomando en cuenta que el núcleo más importante de obras es aquella que data de los cuarenta, el Museo se ocupó de incorporar relatos locales y globales, especialmente aquellos de las vanguardias, para luego dar cuenta de las producciones más contemporáneas, nacionales e internacionales, con un gran hincapié en fotografía y video. Su atención a disciplinas artísticas no tradicionales, como el diseño industrial, permitió que el Museo se integrase a los circuitos de espacios globales contemporáneos, y que sus programas de exposición incluyesen narrativas territoriales diversas. La presencia regional (Sudamericana) está principalmente ligada a los relatos locales, aunque se han producido algunas exhibiciones en tono más regional (por ejemplo, en 2012 se expuso «Corpo Colectivo» con obras de Lygia Clark, y en 2015 se exhibieron trabajos de Laura Lima, también brasileña). Por otro lado, se han producido varias exposiciones internacionales que ligan los relatos del arte locales al circuito global, entre ellos en 2014 se trajo la colección Deutsche Bank, con obras de artistas como Mondrian, Lescher, Freud, Beuys y Baselitz. De modo que se podría afirmar que el Museo funciona con preferencia en los niveles local y global. 
El segundo caso, el Centro Cultural de España en Buenos Aires (CCEBA), es un espacio creado en 1988 bajo el nombre Instituto de Cooperación Iberoamericana (ICl), que funciona a través del financiamiento de una embajada $y$, por lo tanto, se puede suponer que su programa se relaciona a la difusión de actividades relacionadas con el país que representa y con valores sostenidos por su Estado. En cuanto a su misión, está enunciada de la siguiente manera:

Desde 1988, el CCEBA -inicialmente como Instituto de Cooperación Iberoamericana «|C|»- constituye un espacio dedicado al encuentro e intercambio cultural, donde se dan cita destacados creadores iberoamericanos. (...) Entre sus líneas de programación se destacan las actividades formativas, exposiciones, conciertos, proyecciones audiovisuales, encuentros, presentaciones, espacios de debate y publicaciones dedicados a las artes, la gestión cultural, la comunicación, la difusión científica y las nuevas tecnologías ${ }^{3}$.

Como se desprende de su misión, el centro está dedicado a generar proyectos de cooperación entre España y Argentina, aunque también podría decirse que incluye relaciones con otros países de la región. En este sentido, pareciera que en una primera mirada su programa es local, regional y global, en tanto los ciclos, conferencias y actividades suelen poner el foco en los vínculos entre los países miembros del Mercosur y la Unión Europea (de acuerdo con lo afirmado en el Plan Director de la Cooperación Española 2013-2016), y las exposiciones integran artistas y producciones argentinas con la de otros países latinoamericanos y, por supuesto, de España. Una particularidad de este es que además de proyectos que se realizan en sus dos sedes porteñas, el Centro apoya financieramente y promociona actividades y exposiciones en otros centros artísticos. Un ejemplo lo constituyó la exposición Viva España, que se realizó en el Museo de Arte Contemporáneo de Buenos Aires (MACBA) con el apoyo del CCEBA y presentó obras de la artista ibérica Pilar Albarracín. Este caso da cuenta de la inserción del arte global en el escenario local, creando una doble vía de intercambio.

A lo largo de sus años de funcionamiento los programas del centro se fueron modificando para atender a los diferentes y cambiantes objetivos culturales de España y sus relaciones con Argentina y los países de la región Sudame- ricana. Así, se incorporaron especialistas en artes audiovisuales y música, se amplió la mediateca y se redirigieron recursos según los presupuestos establecidos por el área de cultura del gobierno español. En los años noventa, una época particularmente notoria para el entonces $\mathrm{ICl}$, el centro pasó a ser uno de los circuitos obligados del arte contemporáneo del momento, y dialogaba directamente con el espacio de la Galería Ricardo Rojas. Estas dos instituciones apoyaron activamente las producciones de ciertos artistas y constituyeron así dos circuitos que nucleaban a la mayoría de los artistas y críticos de arte contemporáneos. Artistas como Roberto Jacoby o Sebastián Gordín circulaban por ambos, produciendo intercambios interesantes. Además se consolidaron como formadores de tendencias artísticas y, en el caso del Rojas, también curatoriales ${ }^{4}$. Ya entrado el siglo XXI, el CCEBA comenzó a modificar sus programas para incluir actividades diversas y con variadas inscripciones y proveniencias disciplinares y regionales. Actualmente es frecuente la producción de exhibiciones y ciclos de conferencias o proyecciones audiovisuales, encuentros de grupos de estudio de fenómenos no necesariamente artísticos (por ejemplo, en 2011 se produjo un congreso de fenómenos especiales, donde se presentaron diversos especialistas y se discutieron las posibilidades de existencia de vida extraterrestre). Además presentan trabajos y problemáticas de países como Uruguay, Paraguay, Perú o Chile, generando inserciones regionales y globales.

\section{Discusiones preliminares}

En principio, pareciera haber cierto consenso respecto del carácter contingente del arte contemporáneo y la ausencia de predominancia estilística o de modos de producción, que habilita múltiples puntos de vista y formas de hacer. Esa heterogeneidad y la ausencia de una perspectiva teleológica diferencian así al arte contemporáneo del moderno, distancia evidenciada además en la marcada invisibilización de las fronteras de lo artístico en el caso contemporáneo. Es esta misma contingencia la que permite hablar del arte contemporáneo en términos de devenir más que de un producto, y que produce una relación temporal múltiple. En este sentido se produce un efecto de continuo presente que permite reescribir y actualizar tanto el pasado como el futuro, y da lu- 
gar también a diferentes vinculaciones con el tiempo. Así, la instalación por ejemplo produce al menos tres temporalidades: la del interior de la obra, la de circulación del público, y la del vínculo de la instalación con el espacio de exhibición (y con las otras obras en caso de no ser la única presentada).

Al mismo tiempo, esta multiplicidad del presente se puede vincular con la territorial, que a su vez produce multiplicidad identitaria. El desarrollo y la expansión del arte contemporáneo se da a la par de procesos de movimientos globales de capitales que generan la consolidación de ciertas geografías como nuevos centros financieros y, también, artísticos. Esta ampliación no es solamente fronteriza (y de allí que deban surgir nuevas categorías territoriales para acompañar estos cambios, es decir, lo local, regional y global) -aunque allí radica una diferencia con el sistema del arte moderno, en general con pocos centros y movimientos más delimitados- sino también productiva: el arte contemporáneo (que se produce y circula globalmente, en grandes bienales y ferias de arte o en circuitos más pequeños pero interconectados y con presencia en varias geografías) opera sobre los territorios y los procesos de territorialización, y los modifica.

De este modo, la inserción de nuevos actores y de nuevas regionalidades (o localidades) al mapa global del arte contemporáneo pareciera haber logrado una suerte de visibilización de sectores ampliamente ignorados con anterioridad, que se ven afectados por los nuevos procesos de territorialización y desterritorialización y por las prácticas globales. Estas prácticas territoriales también plantean desafíos a la hora de analizar estas incorporaciones, porque la categoría de arte contemporáneo parece englobar la mayoría de la producción actual, y por tanto puede actuar como un homogeneizador. Por ese motivo se planteó en el trabajo la necesidad, no solamente de prestar atención a estos procesos y los modos en los cuales se puede lograr ese señalamiento de producciones provenientes de, por ejemplo, el sur, sino también de relevar algunos casos para entender su funcionamiento y posible alcance. En los ejemplos trabajados, MAMBA y CCEBA, se pudo observar cómo operan los tres niveles de territorialización mencionados (local, regional, global) y el modo en el cual los espacios porteños modulan las cartografías del arte contemporáneo y se incorporan en las lógicas de circulación más global.

Queda un largo camino por recorrer en cuanto al arte contemporáneo y sus formas de producción y circulación, y ciertamente se deberá reflexionar acerca de los modos en los cuales las estéticas y los relatos regionales y locales se pueden pensar en relación a circuitos más amplios. Por otra parte, sería interesante producir investigaciones que permitan analizar las maneras en los cuales los circuitos artísticos contemporáneos configuran una novedad respecto del sistema moderno, y qué efectos generarán a largo plazo estos cambios.

\section{Notas}

1 En http://www.buenosaires.gob.ar/museoartemoderno/historia (fecha de consulta: 11-10-2016).

2 Laura Buccellato (directora del MAMBA entre 1997 y 2013) en comunicación personal, 10-10-2016.

3 Información disponible en http://www.cceba.org.ar/quienes-somos/ (fecha de consulta: 13-10-2016).

4 «La estética del Rojas", por ejemplo, está relacionada al denominado arte light, aquel que producía con elementos desechables y baratos, que parecía superficial, poco denso y desinteresado por la situación argentina.

\section{Bibliografía}

AGAMBEN, G. (2009), «¿Qué es ser contemporáneo?» (trad. Cristina Sardoy), Clarín, 21 de Marzo, s/p. ARANDA, J. et al. (ed.) (2009), «What Is Contemporary Art?», e-flux journal, n. ${ }^{0}$ 11, diciembre, pp. 1-2. - (ed.) (2010), «What Is Contemporary Art?», e-flux journal, n. ${ }^{12}$, enero, pp. 1-3. DELEUZE, G. y GUATTARI, F. (1985), El Anti Edipo. Capitalismo y esquizofrenia, Paidós, Barcelona-Buenos Aires-México. - (1994), Mil mesetas. Capitalismo y esquizofrenia, Pre-Textos, Valencia.

FOSTER, H. (2010), «Contemporary Extracts», e-flux journal, n. ${ }^{\circ}$ 12, enero, pp.1-6. 
GROYS, B. (2009), «Comrads of time», e-flux journal, n. ${ }^{\circ} 11$, diciembre, pp. 1-11.

HAESBAERT, R. (2011), El mito de la desterritorialización. Del «fin de los territorios» a la multiterritorialidad, Siglo XXI, México MEDINA, C. (dir.) (2009), Sur, sur, sur, sur, Séptimo Simposio Internacional de Teoría sobre Arte Contemporáneo, México.

- (dir.) (2010), "Once tesis sobre el arte contemporáneo» (trad. Roberto Jacoby), Ramona. Revista de artes visuales, n. ${ }^{\circ}$ 101, junio-julio, pp. 72-76. Disponible en http://70.32.114.117/gsdl/collect/revista/revistas/ramona101.pdf (fecha de consulta: 22-02-2017).

MOSQUERA, G. (2010), Caminar con el diablo. Textos sobre arte, internacionalismo y culturas, Exit, Madrid.

PARNET, Claire (1996), El abecedario de Gilles Deleuze (entrevista), transmisión por Canal Arté (ed. Por Vidéo Editions Montparnasse). Transcripción disponible en http://imperceptibledeleuze.blogspot.com.ar/search/label/El\%20Abecedario (fecha de consulta: 23-062017).

PLAN DIRECTOR DE LA COOPERACIÓN ESPAÑOLA, 2013-2016, en http://www.aecid.es/Centro-Documentacion/Documentos/Divulgaci\%C3\%B3n/iv_plan_directorce_2013-2016_final2.pdf (fecha de consulta: 10-07-2017).

SCHAEFFER, J.M. (2013), «Experiencia estética: placer y conocimiento», en Boletín de estética, n. ${ }^{25}$, pp. 5-34. Disponible en http://www. boletindeestetica.com.ar/wp-content/uploads/Boletin-de-Estetica-25.pdf (fecha de consulta: 22-02-2017).

SMITH, T. (2012), ¿Qué es el arte contemporáneo?, Siglo XXI, Buenos Aires. 
